\title{
MicroRNA-340 inhibits prostate cancer cell proliferation and metastasis by targeting the MDM2-p53 pathway
}

\author{
KAI HUANG, YUXIN TANG, LEYE HE and YINGBO DAI
}

Department of Urology, The Third Xiangya Hospital of Central South University, Changsha, Hunan 410013, P.R. China

Received July 16, 2015; Accepted September 4, 2015

DOI: $10.3892 /$ or.2015.4458

\begin{abstract}
An increasing number of studies have demonstrated the important role of microRNAs (miRNAs) in modulating cancer progression and metastasis, but the mechanisms by which miRNAs regulate prostate cancer $(\mathrm{PCa})$ tumorigenesis remain poorly understood. In the present study, we found that miR-340 may act as a tumor suppressor based on our finding that it was significantly downregulated in PCa tumor tissues and cell lines. Moreover, the expression of miR-340 was found to be correlated with the inhibition of cell proliferation, migration and invasion in vitro, and had a suppressive effect on tumor growth in a xenograft mouse model as well. The suppressive effect of miR-340 overexpression was observed in cell lines DU145 and BPH-1 which express wild-type (WT) p53. However, in the p53-null PC-3 cell line, the suppressive effect was not found, indicating that miR-340 may play a critical role in the p53 pathway. Further investigation revealed that mouse double minute 2 (MDM2), an important regulator of p53, was targeted by miR-340 through the direct binding to the 3'UTR of MDM2, which inhibited MDM2 translation. In addition, miR-340 expression stabilized p53 protein levels which caused an increase in p21 expression but a decrease in the anti-apoptotic protein, BCL-2, in the p53 WT cell lines. Moreover, the miR-340-mediated inhibition of cell progression was mitigated by re-expressing MDM2 in the stable miR-340-overexpressing PCa cell line, which harbors WT p53. Our findings suggest that miR-340 may function as a novel tumor suppressor in PCa through the MDM2-p53 pathway by directly targeting MDM2, which may be a promising miRNA-targeted therapy for PCa.
\end{abstract}

Correspondence to: Dr Yuxin Tang, The Third Xiangya Hospital of Central South University, 138 Tongzipo Road, Changsha, Hunan 410013, P.R. China

E-mail: yuxinttt@sina.cn

Key words: miR-340, prostate cancer, MDM2, p53, microRNAs

\section{Introduction}

Prostate cancer (PCa) is the most common newly diagnosed cancer in the United States in 2015 and remains the second leading cause of cancer-related mortality, trailing only lung cancer (1). Metastasis, a highly complex, multistep biological process that involves several important events and molecular factors, is a major cause of death in patients with PCa (2). Although various oncogenes and tumor-suppressor genes have been reported in a previous research to play important roles in the process of metastasis, the molecular mechanisms of prostate carcinogenesis remain unclear (3). Therefore, a better understanding of the underlying molecular mechanisms of metastasis is essential for the development of novel and effective therapies against $\mathrm{PCa}$.

miRNAs are a large class of endogenous small non-coding RNAs that are 22 nucleotides long. miRNAs regulate gene expression by binding to the 3 ' untranslated regions (UTRs) of target mRNAs in a partially complementary manner, which leads to mRNA degradation or suppression of translation (5).

An increasing number of studies have shown that miRNAs may not only play a significant role in PCa progression $(6,7)$ such as proliferation (7), the cell cycle (8), apoptosis (9), invasion and metastasis (10-12) but also function as tumor suppressors or oncogenes depending on the genes that they regulate (13).

Previous studies have shown that the proliferation, invasion and metastasis of PCa involve various genomic alterations. Dubovenko et al (14) reported a contribution effect of the RAS-RAF-mitogen-activated protein kinase (MAPK) signaling pathway in PCa development. Qiu et al (15) found that the low expression of cyclin-dependent kinases (CDKs) (CDK2, CDK4, CDK6) downregulated by sodium butyrate play an important role in the G1 arrest of PCa cells. Meanwhile, the alterative expression of cell cycle regulatory proteins such as cyclin was found to be related to PCa cell growth as well (16).

Previous studies have found that miR-340 plays critical roles in the biological processes of cancers. Li et al (17) demonstrated that miR-340 inhibits glioblastoma cell proliferation by directly targeting CDK6, cyclin D1 and cyclin D2. Furthermore, Fernandez et al found that miR-340 was identified as a novel tumor suppressor in non-small cell lung cancer (18), and also inhibits the tumorigenic phenotype of melanomas by regulating the RAS-RAF-mitogen-activated protein kinase (MAPK) signaling pathway (19). However, the 
role and underlying mechanisms of miR-340 in PCa remains unclear. In the present study, for the first time, we showed that miR-340 acts as a tumor suppressor in PCa based on the finding that miR-340 inhibited PCa cell proliferation, migration and invasion by targeting the MDM2 proto-oncogene and by inhibiting MDM2-dependent p53 inhibition. Our findings suggest the potential efficacy of a therapy involving the overexpression of miRNA-340 to treat human PCa.

\section{Materials and methods}

Tissue specimens and cell lines. PCa tissue specimens and corresponding non-tumor samples were collected from 50 PCa patients who underwent radical prostatectomy at the Third Xiangya Hospital of Central South University from 2010 to 2013. Written informed consent was obtained from all of the patients, and this study was conducted in accordance with the ethical standards set forth by the Institutional Committee, approved by the IRB of Third Xiangya Hospital, Central South University, no. 2015-S147. Human PCa cell lines (DU145 and PC-3) and normal prostate epithelial cells (RWPE-1) were purchased from the Cell Bank at the Chinese Academy of Sciences. Human benign prostate hyperplasia (BPH-1) cells were obtained from the American Type Culture Collection (ATCC; Manassas, VA, USA). DU145, PC-3 and BPH-1 cells were grown in Dulbecco's modified Eagle's medium (DMEM), F-12 and RPMI-1640 medium (Invitrogen, Carlsbad, CA, USA), respectively, and RWPE-1 cells were maintained in K-SFM containing $0.05 \mathrm{mg} / \mathrm{ml}$ bovine pituitary extract (BPE) and $5 \mathrm{ng} / \mathrm{ml}$ epidermal growth factor (EGF). All media were supplemented with $10 \%$ fetal bovine serum and a $1 \%$ antibiotic/antimycotic solution (Sigma, St. Louis, MO, USA). All cell lines were incubated at $37^{\circ} \mathrm{C}$ in the presence of $5 \% \mathrm{CO}_{2}$.

Plasmids and cell transfection. The miR-340 mimic/inhibitor and the scrambled control were purchased from GenePharma (Shanghai, China). The pcDNA3.1-MDM2 plasmid was constructed by YRBIO (Changsha, China). The cells were seeded in 6- or 96-well plates at 30\% confluency $24 \mathrm{~h}$ prior to transfection, and the cells were then transfected with the miR-340 mimic/inhibitor or the controls using Lipofectamine 2000 reagent (Invitrogen) according to the manufacturer's protocol.

Quantitative real-time PCR. miRNA and total RNA were extracted from the cells or tissues using the miRNeasy Mini kit (Qiagen, Valencia, CA, USA) and TRIzol reagent (Invitrogen), respectively. Reverse transcription was performed using a miScript II RT kit (Qiagen), and qRT-PCR was performed on a CFX96 real-time thermocycler (BioRad, Hercules, CA, USA) using SYBR Premix Ex Taq kits (Takara, Dalian, China). All the primer sets are listed in Table I. Each assay was performed in triplicate. U6 and GAPDH were used as endogenous controls, and relative expression was calculated using the $2^{-\Delta \Delta C T}$ method.

Cell proliferation assay. A total of $10^{4}$ cells/well were seeded in 96-well plates in triplicate. At 24, 48 and $72 \mathrm{~h}$ after transfection, cell proliferation was assessed using the MTT reagent
(Roche, Indianapolis, IN, USA), and the absorbance was measured for each time-point at a wavelength of $450 \mathrm{~nm}$ using a 96 -well plate reader.

In vitro cell migration and invasion assays. Cells were resuspended in serum-free medium before assessing cell migration and invasion capacity. A total of $5 \times 10^{4}$ cells were seeded into the top chamber of Transwell inserts (Corning, Tewksbury, MA, USA) for the migration assay, and $1 \times 10^{5}$ cells were plated in the upper chamber precoated with Matrigel (BD Biosciences) for the invasion assay. Medium containing $10 \%$ FBS was added to the bottom chamber, and the plates were incubated for $24 \mathrm{~h}$. After incubation, the inserts containing cells adherent to the lower membrane were fixed and stained with $20 \%$ methanol and $0.2 \%$ crystal violet, respectively. The stained cells were imaged and counted.

Flow cytometric analysis for apoptosis. Cells were collected after transfection with the miR-340 mimic, inhibitor or control, and the percentage of apoptotic cells was measured by flow cytometry using an Annexin V-FITC/PI double-staining kit and a FACSCalibur flow cytometer (Beckman Instruments, Fullerton, CA, USA). Five independent assays were performed.

Western blot analysis. Cells were harvested, and protein was extracted using RIPA buffer. The bicinchoninic acid method was used to determine protein concentration. A total of $25 \mu \mathrm{g}$ of protein was separated by SDS-PAGE and transferred to PVDF membranes (Millipore, Billerica, MA, USA) to be probed with the following antibodies: mouse anti-MDM2 (1:1,000), mouse anti-p21 (1:800) and mouse anti-BCL2 (1:500) (Abcam, Cambridge, UK). HRP-conjugated goat anti-mouse antibody (1:1,000; Cell Signaling Technology, Danvers, MA, USA) was used as the secondary antibody. Signals were detected using ECL Western Blotting Substrate (Thermo Fisher Scientific, USA) and were quantified using Image $\mathbf{J}$ software.

Generation of stable miR-340-overexpressing PCa cells. miR-340 overexpression or control lentiviral vectors were purchased from YRBIO and were used to infect the PCa cells (DU145 and PC-3) for $72 \mathrm{~h}$. Cells were selected with $2 \mu \mathrm{g} / \mathrm{ml}$ puromycin at $\sim 2$ weeks.

Dual-luciferase reporter assay. Luciferase reporter vectors containing the seed sequence for miR-340 corresponding to the wild-type (WT) 3'UTR of MDM2 and a mutated version of the 3'UTR of MDM2 containing mutations within the core binding site for miR-340 were constructed. HEK-293 cells were seeded into 96-well plates and were co-transfected with WT 3'UTR MDM2 or mutant 3'UTR MDM2 and miR-340 or a control scrambled sequence using Lipofectamine 2000. Firefly and Renilla luciferase activities were determined using the Dual-Luciferase reporter system (Promega, Madison, WI, USA) $48 \mathrm{~h}$ after transfection. The experiments were performed independently in triplicate, and the data are presented as the mean ratio of Renilla activity/firefly luciferase activity.

Tumorigenesis assays. A cell suspension of $2 \times 10^{6}$ cells/mouse was injected subcutaneously into the flanks of 4- to 6-week-old female nude mice ( $n=5 /$ group). The tumor volume was calcu- 
Table I. Primer sequences.

\begin{tabular}{|c|c|c|}
\hline Name & Primer & Sequence $\left(5^{\prime}-3^{\prime}\right)$ \\
\hline \multirow[t]{3}{*}{ miRNA-340 } & Reverse transcription primer: & GTCGTATCCAGTGCGTGTCGTGGAGTCGGCAATTGCACTGGATACGACAATCAG \\
\hline & Forward primer: & GCGGTTATAAAGCAATGAGA \\
\hline & Reverse primer: & GTGCGTGTCGTGGAGTCG \\
\hline \multirow[t]{3}{*}{ U6 snRNA } & Reverse transcription prime: & AAAATATGGAACGCTTCACGAATTTG \\
\hline & Forward primer: & CTCGCTTCGGCAGCACATATACT \\
\hline & Reverse primer: & ACGCTTCACGAATTTGCGTGTC \\
\hline \multirow[t]{2}{*}{ GAPDH } & Forward primer: & CCAGGTGGTCTCCTCTGA \\
\hline & Reverse primer: & GCTGTAGCCAAATCGTTGT \\
\hline \multirow[t]{2}{*}{ MDM2 } & Forward primer: & GCAGGGCTTATTCCTTTTCTTTA \\
\hline & Reverse primer: & CATTGAACCTTGTGTGATTTGTC \\
\hline \multirow[t]{2}{*}{ p21 } & Forward primer: & TCCTCTAGCTGTGGGGGTGA \\
\hline & Reverse primer: & GAAGGTCGCTGGACGATTTG \\
\hline \multirow[t]{2}{*}{ BCL-2 } & Forward primer: & CGCCCTGTGGATGACTGAGTA \\
\hline & Reverse primer: & CCTCAGCCCAGACTCACATCA \\
\hline
\end{tabular}
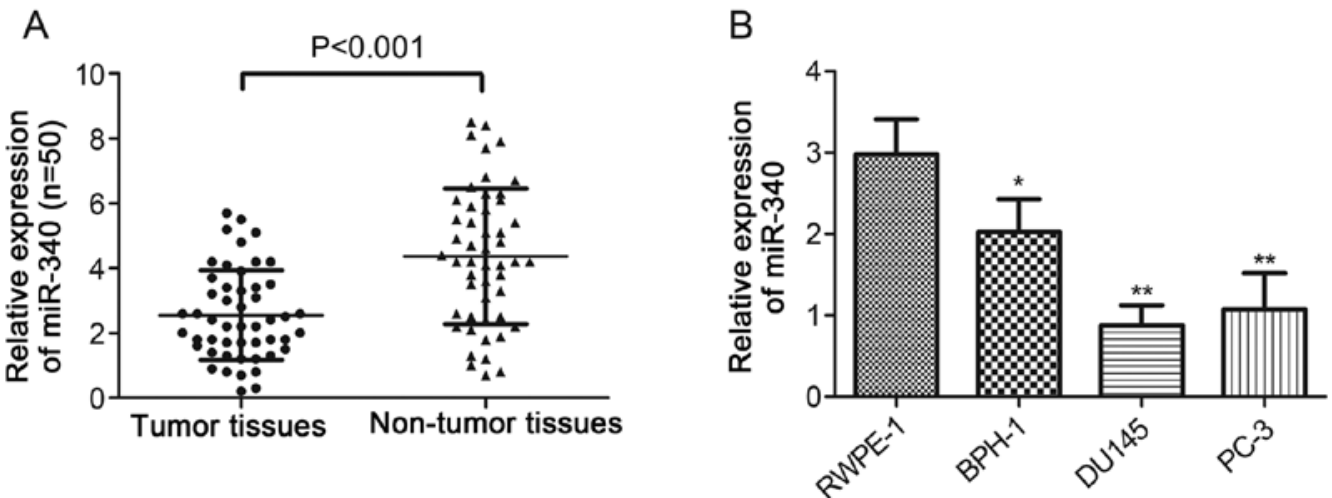

Figure 1. miR-340 is downregulated in PCa tumor tissues and cells. (A) Dot plots showing miR-340 expression levels in PCa tissues compared with adjacent non-tumor tissues. $\mathrm{P}<0.001$ vs. non-tumor tissues. (B) Histogram showing miR-340 expression in three cell lines derived from PCa and one normal prostate cell line. ${ }^{*} \mathrm{P}<0.05$ and ${ }^{* *} \mathrm{P}<0.01$. Data are expressed as the means $\pm \mathrm{SD}$.

lated twice a week using microcalipers, and the study was terminated 30 days after implantation. The tumor tissues were snap frozen in liquid nitrogen and stored at $-80^{\circ} \mathrm{C}$ for subsequent analysis.

Statistical analysis. All statistical analyses were performed with SPSS 13.0 (SPSS Inc., Chicago, IL, USA). The data values were presented as the mean \pm SEM. Mean value differences between two groups were analyzed by the two-tailed Student's t-test, and the means of three groups were compared with one-way ANOVA. P-value $<0.05$ was considered to indicate a statistically significant difference.

\section{Results}

Expression of miR-340 is significantly downregulated in $\mathrm{PCa}$ cells and tissues. To evaluate the role of miR-340 in PCa progression, 50 pairs of tumor and adjacent non-tumor tissues obtained from PCa patients were analyzed. The real-time
PCR results indicated that miR-340 expression was significantly downregulated in the PCa tissues when compared with that noted in the matched normal tissues $(\mathrm{P}<0.001$, Fig. 1A). Furthermore, the miR-340 levels were substantially downregulated both in the $\mathrm{PCa}$ cell lines $(\mathrm{P}<0.01)$ and $\mathrm{BPH}-1$ cells $(\mathrm{P}<0.05)$ compared with the level in the normal prostate epithelial cells (RWPE-1), respectively (Fig. 1B). The downregulation of miR-340 both in PCa cells and tissues indicates that miR-340 may play an important role in $\mathrm{PCa}$.

miR-340 suppresses PCa cell proliferation, migration and invasion as well as induces apoptosis. To investigate the function of miR-340 in PCa tumorigenesis, two PCa cell lines (DU145 and PC-3) and BPH-1 cells were transfected with a commercially available miR-340 mimic or inhibitor. The levels of miR-340 were significantly increased upon transfection with the miR-340 mimic and were decreased upon transfection with the miR-340 inhibitor $(\mathrm{P}<0.05$, Fig. $2 \mathrm{~A})$. Upregulation of miR-340 resulted in a significant decrease in cell proliferation 

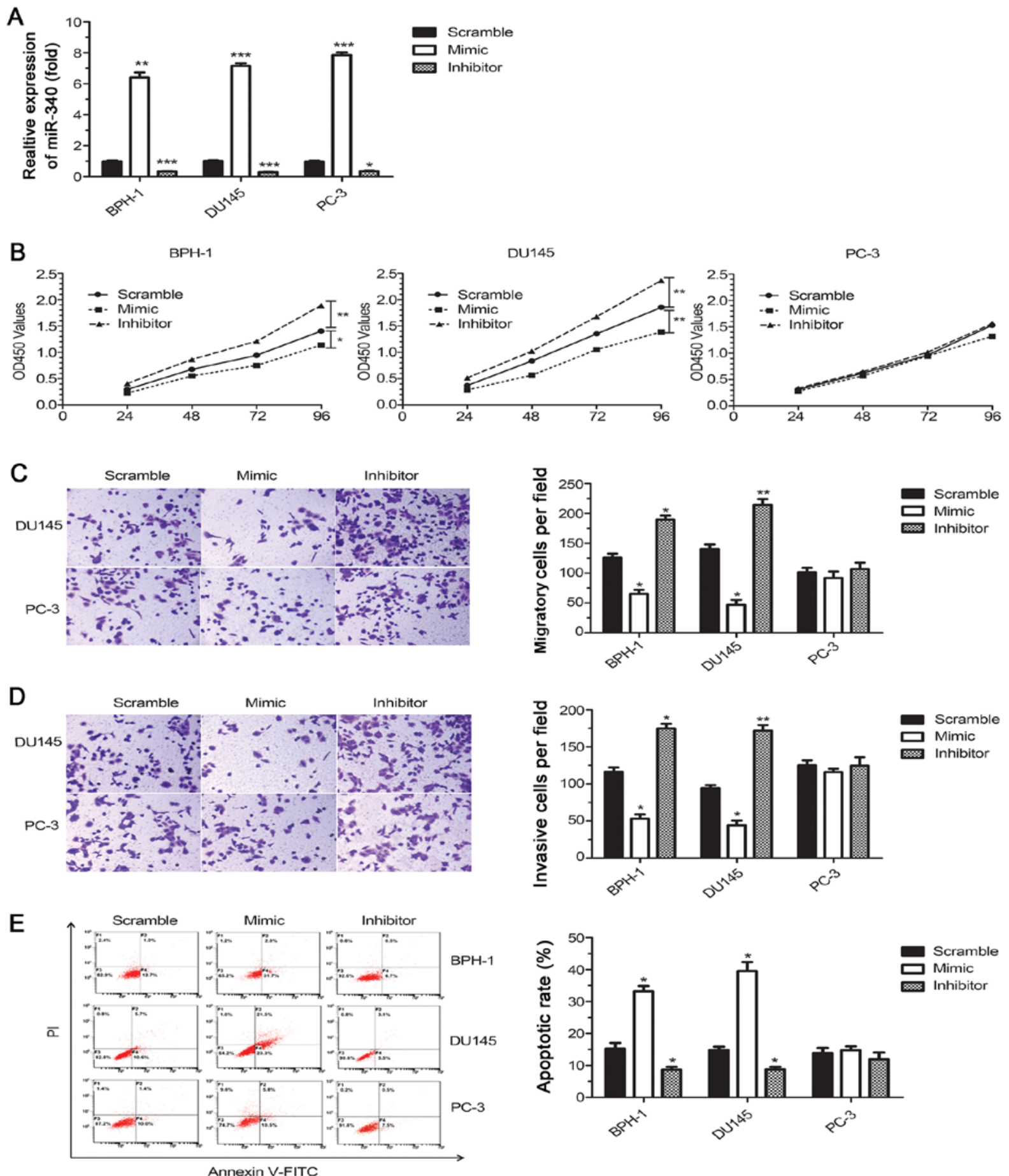

Figure 2. miR-340 inhibits proliferation, migration and invasion and promotes apoptosis in PCa cells. (A) Relative miR-340 expression levels were determined by real-time PCR in cells that were transfected with $20 \mathrm{nmol} / 1 \mathrm{miR}-340$ mimics, inhibitors or the scrambled RNA control. Results were normalized to U6 snRNA. (B) Proliferative ability was measured by the MTT assay at different time-points. (C and D) The migratory and invasive capacities of the PCa cells were determined by Transwell analysis $(n=5)$. Representative images of migrated/invaded cells are shown. (E) Apoptosis was measured by flow cytometry, and the graphs show the percentage of apoptotic cells $(\mathrm{n}=5)$. All data are expressed as the means \pm SD. ${ }^{*} \mathrm{P}<0.05,{ }^{* * *} \mathrm{P}<0.01$ and ${ }^{* * * *} \mathrm{P}<0.001$ vs. cells transfected with the scrambled control.

in the two p53 WT cell lines (DU145 and BPH-1) but not in the PC-3 cells, which lack WT p53. Consistently, downregulation of miR-340 increased cell proliferation in the DU145 and $\mathrm{BPH}-1$ cells $(\mathrm{P}<0.05)$ but had no effect on the PC-3 cells $(\mathrm{P}>0.05$, Fig. $2 \mathrm{~B})$. Furthermore, the overexpression of miR-340 reduced cell migration and invasion in the cell lines expressing WT p53 ( $<<0.05$, Fig. $2 \mathrm{C}$ and D). Flow cytometric results showed a sharp increase in the percentage of apoptotic cells for miR-340 mimic recipients and a decrease in apoptosis in the miR-340 inhibitor recipients compared with the scrambled control $(\mathrm{P}<0.05$, Fig. 2E). Notably, these effects were absent in the PC-3 cells, which lack WT p53, indicating that miR-340 may interact with the p53 pathway.

miR-340 directly targets MDM2 to regulate the MDM2-p53 pathway. To determine the mechanism by which miR-340 
A

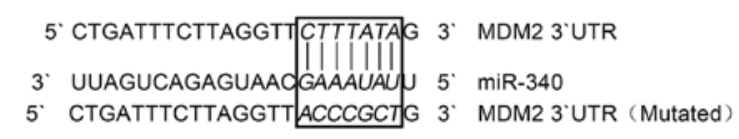

C

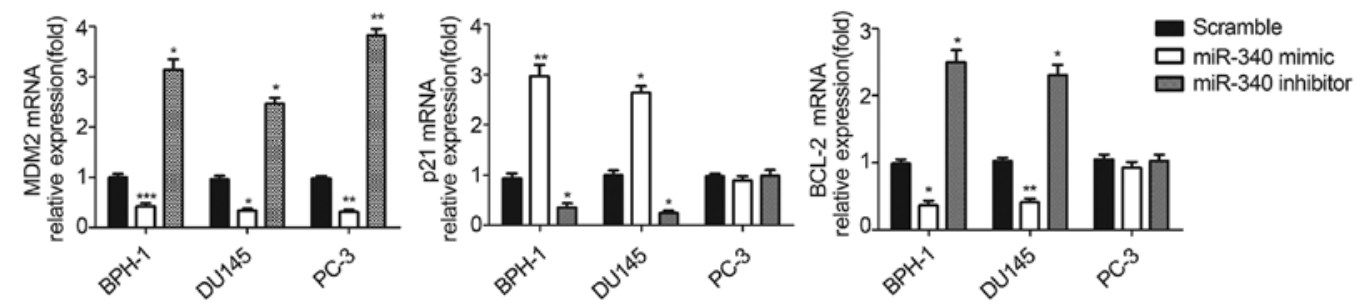

D
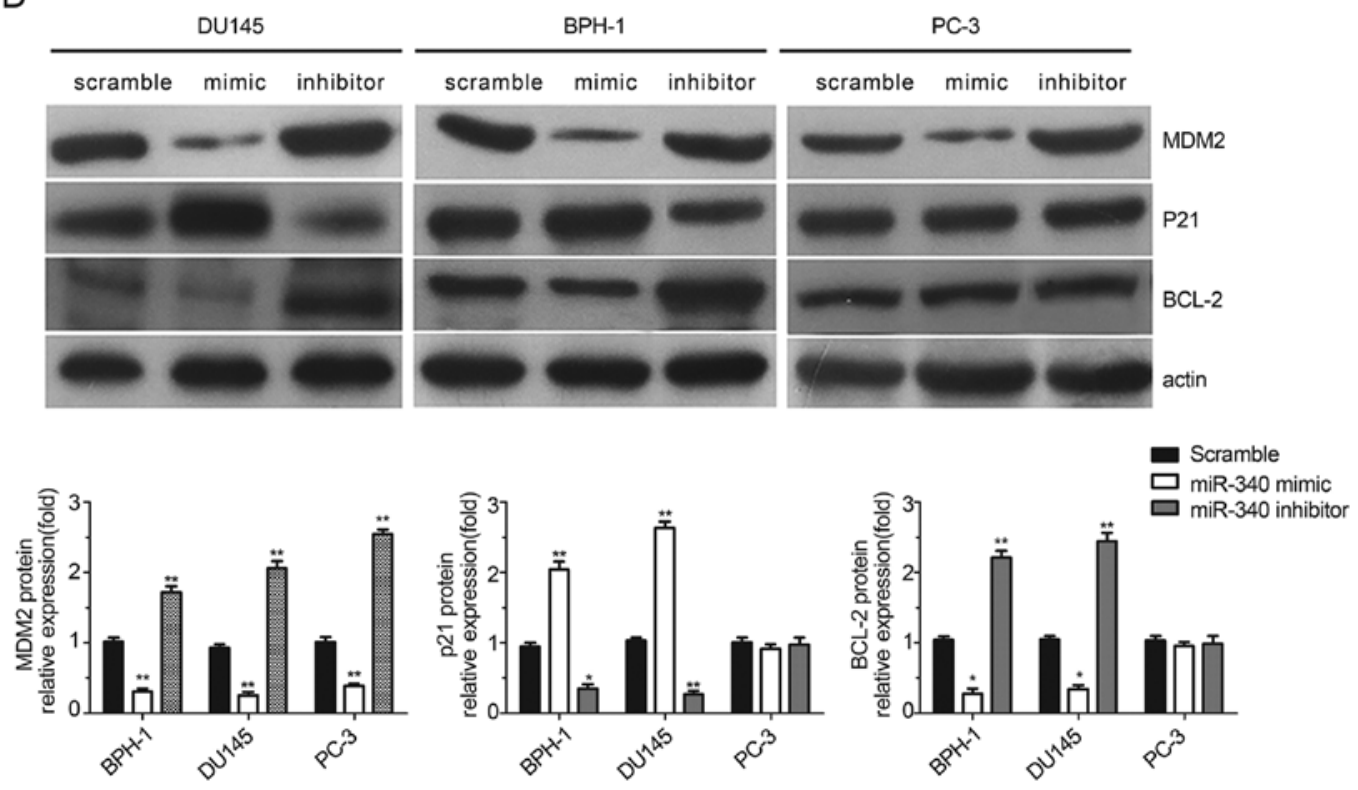

E

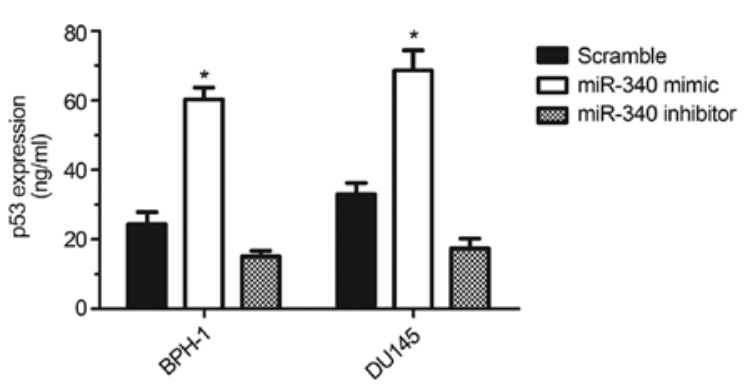

Figure 3. miR-340 directly targets MDM2 and regulates the p53 pathway. (A) The predicted core binding site of miR-340 within the 3'UTR of MDM2 and the mutated 3'UTR of MDM2 are shown. (B) A dual-luciferase reporter assay showed that miR-340 was able to bind to the wild-type 3'UTR but not the mutated 3'UTR of MDM2, which suggests that MDM2 is a direct target of miR-340. (C and D) The mRNA and protein levels of MDM2, p21 and BCL-2 in PCa cells transfected with the miR-340 mimics, inhibitors or the scrambled control were measured by real-time PCR and western blot analyses $(n=3)$. (E) p53 expression levels in cells transfected with the miR-340 mimics or inhibitors were analyzed by ELISA $(n=5)$. All data are expressed as the means \pm SD. ${ }^{*} \mathrm{P}<0.05,{ }^{* *} \mathrm{P}<0.01$ vs. cells transfected with the scrambled control.

affects the functions of cancer cells and interacts with $\mathrm{p} 53$, we predicted miR-340 targets by TargetScan and focused on target genes encoding proteins involved in the p53 pathway. Mouse double minute 2 (MDM2), an important regulator of the p53 tumor suppressor, was found to be a novel target candidate of miR-340 as the 4293-4299 position of the MDM2 3'UTR was complementary to the seed sequence of miR-340. To validate this potential direct interaction, we constructed luciferase 
A

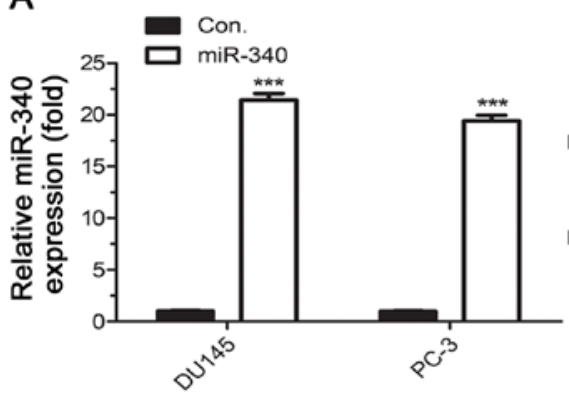

C

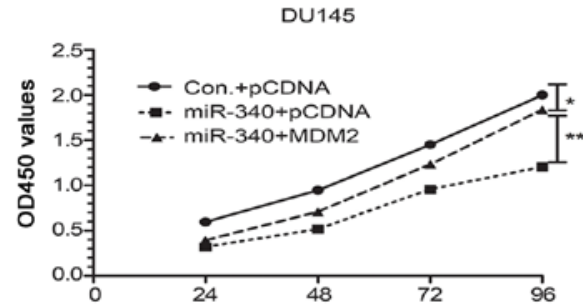

B
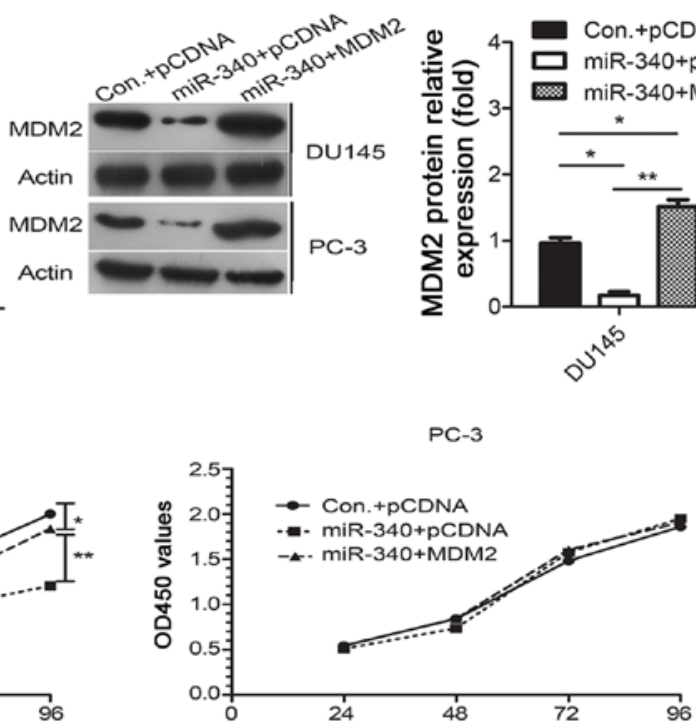

D
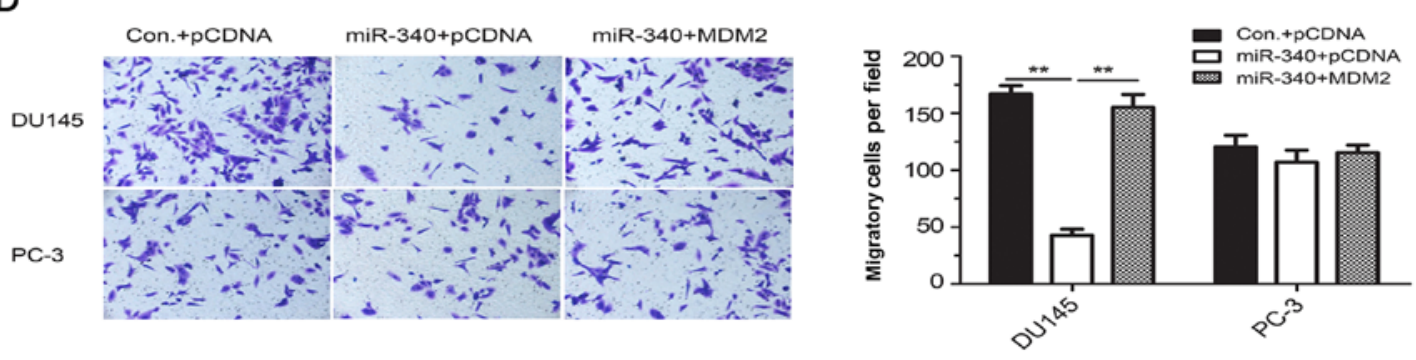

$E$
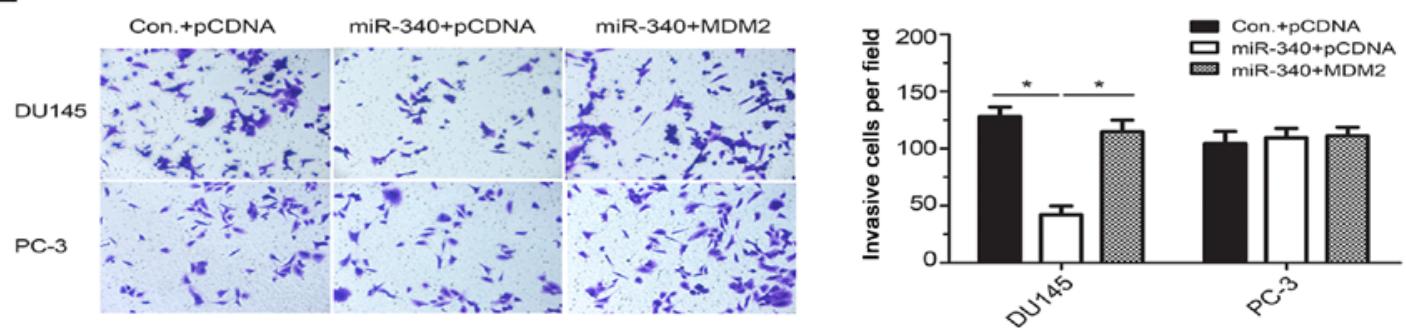

Figure 4. MDM2 reverses the effects of miR-340 on PCa cells. (A) The levels of miR-340 expression were upregulated in the stable miR-340-overexpressing cells. (B) MDM2 expression in the miR-340-overexpressing PCa cells or control cells transfected with 1.0 $\mu \mathrm{g}$ of pcDNA3.1-MDM2 vector or pcDNA3.1 empty vector was analyzed by western blot analysis, and representative bands are shown. (C) Proliferative ability was measured using the MTT assay ( $\mathrm{n}=5$ ). (D and E) Transwell analysis of DU145 and PC-3 cells transfected with different combinations of expression constructs ( $\mathrm{n}=5$ ). The number of migrated or invaded cells per field is shown on the right, and representative images of migratory/invasive cells are shown on the left ( $\mathrm{n}=5$ ). All data are expressed as the means $\pm \mathrm{SD} .{ }^{*} \mathrm{P}<0.05,{ }^{* *} \mathrm{P}<0.01$ and ${ }^{* * *} \mathrm{P}<0.001$ vs. the control cells.

reporter vectors containing the WT and mutant (Mut) 3'UTR of MDM2 (Fig. 3A). Dual-luciferase reporter assays showed a statistically significant decrease (63\% reduction) in the luciferase activity of 3'UTR MDM2 $(\mathrm{P}<0.05)$, but no changes were found in the luciferase activity of the mutant $(\mathrm{P}>0.05$, Fig. $3 \mathrm{~B})$. In addition, all cell lines transfected with the miR-340 mimics showed a decrease both in MDM2 mRNA and protein levels as measured by real-time PCR and western blot analyses, respectively $(\mathrm{P}<0.05$, Fig. $3 \mathrm{C}$ and $\mathrm{D})$. These finding showed that miR-340 may directly target the 3'UTR of MDM2 and have an effect on the regulation of its expression.

To confirm the effect of miR-340 on p53 expression, we measured p53 levels in cell lysates by ELISA. A significant increase in p53 expression was detected after miR-340 upregulation in the BPH-1 and DU145 cells, which harbor WT p53 ( $<<0.05$, Fig. 3E). To determine whether the antitumor activity of miR-340 is dependent on p53, we analyzed the mRNA and protein levels of $\mathrm{p} 21^{\mathrm{WAF} / / \mathrm{CIP} 1}$, a cyclin-dependent kinase inhibitor that functions as a p53-dependent cell cycle checkpoint. Our analysis revealed a statistically significant increase in p21 levels in cells treated with the miR-340 mimics and a decrease in p21 expression in cells treated with the miR-340 inhibitors $(\mathrm{P}<0.05)$. Furthermore, the expression of the anti-apoptotic gene, BCL-2, exhibited the opposite trend in the WT p53 cell lines (Fig. 3C and D). Consistent with a p53-dependence, the mRNA and protein levels of p21 and BCL-2 remained unchanged in the PC-3 p53-null cells despite clear changes in MDM2 expression 
A
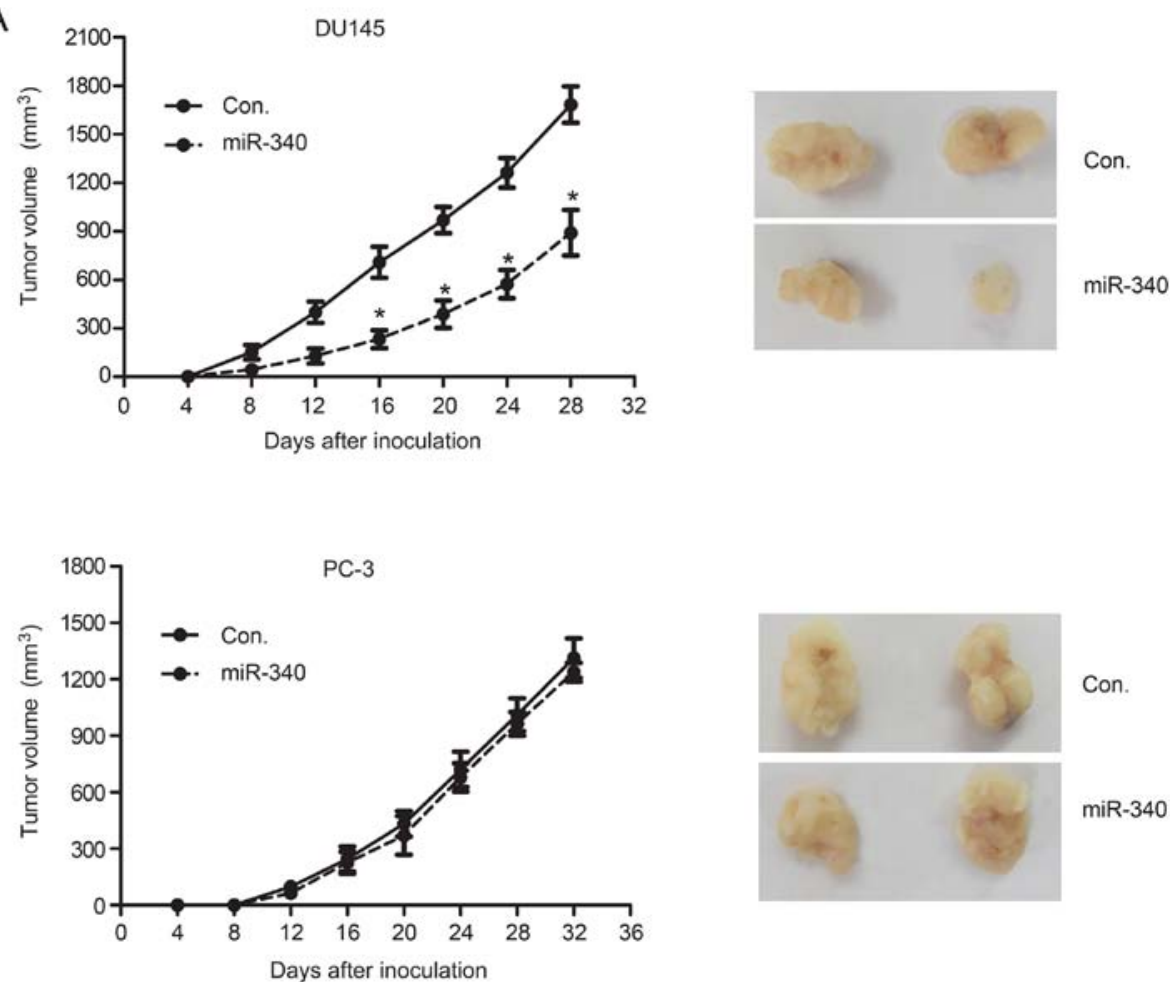

B

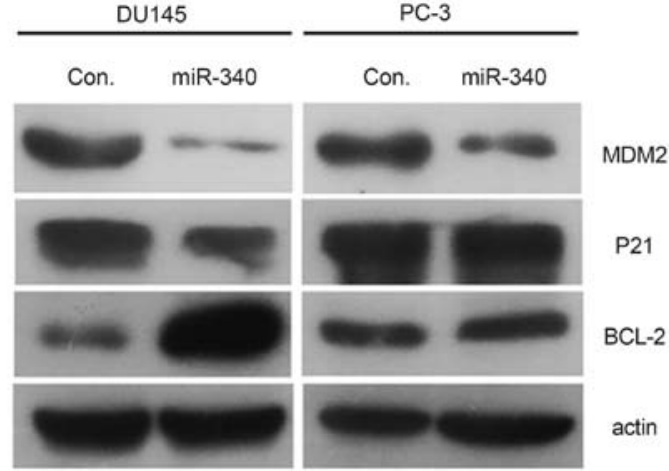

Figure 5. miR-340 inhibits xenograft tumor growth in mice. (A) The tumor growth curves of nude mice subcutaneously injected with miR-340-overexpressing prostate cells (DU145 and PC-3) and the control cells (n=5/group). Representative images of tumor size are shown to the right. (B) Expression of MDM2, BCL-2 and p21 in subcutaneous tumors expressing miR-340 or control miRNA was detected by western blot analysis. Representative bands are shown. All of the data are expressed as the means $\pm \mathrm{SD}$. ${ }^{*} \mathrm{P}<0.05$ vs. the control.

levels (Fig. 3C and D). These results suggest that miR-340 may play a tumor-suppressive role in the presence of functional p53 protein by targeting MDM2.

$M D M 2$ reverses miR-340-induced inhibition of growth, migration and invasion in PCa cells. Stable miR-340-overexpressing PCa cells were generated using lentiviral vectors, and all the stable cells expressed significantly increased levels of miR-340 and significantly decreased levels of MDM2 protein compared to their corresponding controls $(\mathrm{P}<0.05$, Fig. $4 \mathrm{~A}$ and $\mathrm{B})$. Moreover, a series of in vitro assays revealed an inhibition of cell growth and a marked decrease in migration and invasion in these stable miR-340-overexpressing cells. Furthermore, rescue experiments showed that re-expression of MDM2 by transfection with the pcDNA3.1-MDM2 plasmid mitigated the inhibition of proliferation, migration and invasion in the miR-340-overexpressing DU145 cells (Fig. 4C-E). As expected, these effects were abolished in the miR-340-overexpressing PC-3 cells lacking WT p53, which was consistent with the data obtained from the transient transfection experiments in the PC-3 cells. Overexpression of MDM2 alleviated the effects induced by miR-340 in the WT p53 cells but not in the p53-null cells, which suggests that functional p53 protein is essential for the tumor suppressive activity of miR-340.

miR-340 inhibits xenograft tumor growth. To evaluate the tumor-suppressive effects of miR-340 in vivo, we subcutaneously injected stable miR-340-overexpressing PCa cells into nude mice. A significant suppressive effect on the growth of miR-340-overexpressing tumors was observed in the DU145 cells (WT p53), but these effects were not observed in the PC-3 cells lacking WT p53 protein (Fig. 5A). Although the 
protein level of MDM2 in the tumor tissues was reduced in all miR-340-overexpressing cells, upregulation of p21 protein and downregulation of BCL-2 protein were observed in the WT p53 cells but not in the p53-null cells compared with the control ( $\mathrm{P}>0.05$, Fig. 5B), which was similar to the in vitro results. These results support the tumor-suppressive role of miR-340 and underscore the importance of the MDM2-p53 pathway in the miR-340-induced tumor suppressive effects using an in vivo xenograft model.

\section{Discussion}

miRNAs are involved in many critical biological processes such as tumorigenesis and cancer metastasis. Growing evidence indicates that miRNAs can act as ideal molecular targets for cancer diagnosis, prognosis and therapy in many types of cancers (20-22). Various miRNAs have been used to detect cancer and to develop miRNA-based therapeutic strategies in PCa (23-25). To explore different strategies for cancer therapeutics, more studies are needed to identify novel and valuable miRNAs and further, to investigate the role and molecular mechanisms of these miRNAs in PCa tumorigenesis.

Previous findings have confirmed that miR-340 suppresses tumor cell migration and invasion in non-small cell lung cancer (17), melanomas (18), breast cancer (26) and osteosarcomas (27). In the present study, we found that miR-340 is a potential novel tumor suppressor associated with $\mathrm{PCa}$ which was partly consistent with previous studies. Although there is evidence that miR-340 is downregulated in several types of cancers, the role of miR-340 and the underlying mechanisms of action in the tumorigenesis of $\mathrm{PCa}$ have not yet been reported. Our results demonstrated for the first time that miR-340 is significantly downregulated in PCa cell lines and tissues. Overexpression of miR-340 decreased cell proliferation, migration and invasion. It promoted apoptosis in vitro, and inhibited tumor growth in vivo as well. Our findings indicate that miR-340 may be associated with PCa tumorigenesis, which could be considered as a potential biomarker for PCa. The inhibitory effect of miR-340 overexpression on cell migration and invasion was only observed in p53 WT cells such as DU145 and BPH-1 cells, but not in PC-3 p53-null cells which suggests that miR-340 may function in a p53-dependent manner. Using in silico algorithms and functional analyses, we identified MDM2, a p53 E3 ubiquitin ligase (28) and principal negative regulator of the expression and function of p53 $(29,30)$, as a miR-340 target. A core complementary sequence of miR-340 was identified in the 3'UTR of MDM2 as miR-340 overexpression suppressed luciferase activity and downregulated MDM2 mRNA and protein levels in cells. These results indicated that miR-340 functions as a tumor suppressor, in part, by targeting the MDM2 oncogene in PCa.

MDM2 plays an important role in the progression of several cancers $(31,32)$, and MDM2 expression is positively correlated with tumor volume and the proliferative index of PCa cells (33). Additionally, MDM2 has been suggested as an intriguing therapeutic target for PCa therapy, and MDM2 inhibitors, such as second-generation antisense oligos, may have a broad spectrum of antitumor activity in human cancers (34). MDM2 promotes the degradation of p53 by ubiq- uitination, functioning in a negative feedback regulatory loop of p53, which regulates cell growth and apoptosis. In our study, the relationship between miR-340 and p53 was determined, and the role of miR-340 in the regulation of migration and invasion was dependent on the p53 status, as restoration of functional MDM2 reversed the effects induced by miR-340 expression in WT p53 cells but not in p53-null cells. Similar data were obtained in vivo, whereby significant inhibition of tumor xenograft growth was detected with miR-340 stably transfected DU145 cells but not with miR-340 stably transfected p53-null PC-3 cells. These data were consistent with previous studies showing that miR-660 and miR-18b also hinder cell proliferation and metastasis only in the presence of WT p53 $(32,35)$.

Furthermore, our in vivo and in vitro WT p53 miR-340 overexpression models showed the upregulation of $\mathrm{p} 21^{\mathrm{WAF} 1 /}$ CIP and the downregulation of MDM2 and BCL-2, which were accompanied by substantial arrest of cell proliferation and induction of apoptosis in PCa cells. Although smallmolecule antagonists of the p53-MDM2 interaction such as nutlins $(36,37)$ or MDM2 inhibitors $(4,38)$, have been developed in the past few years, the development of novel molecular targeting methods for MDM2 is a remaining need for cancer therapy. Our data suggest that the restoration of $\mathrm{p} 53$ activity by targeting MDM2 using miR-340 may be an effective approach in PCa therapy.

However, there were some limitations to our study. The correlation between miR-340 and MDM2 levels in PCa tissues should be examined, and additional cell types should be tested. Moreover, miR-340 likely targets other genes and participates in other signaling pathways in addition to the MDM2 pathway for the regulation of cell growth in PCa.

In conclusion, our findings showed that miR-340 may be a novel tumor-suppressive miRNA and has a regulatory effect on the MDM2-p53 pathway, which could be considered as a basis for the development of miRNA-targeted therapies for prostate and other cancers.

\section{References}

1. Siegel RL, Miller KD and Jemal A: Cancer statistics, 2015. CA Cancer J Clin 65: 5-29, 2015.

2. Valastyan S and Weinberg RA: Tumor metastasis: Molecular insights and evolving paradigms. Cell 147: 275-292, 2011.

3. Xu L, Wang Z, Li XF, He X, Guan LL, Tuo JL, Wang Y, Luo Y, Zhong HL, Qiu SP, et al: Screening and identification of significant genes related to tumor metastasis and PSMA in prostate cancer using microarray analysis. Oncol Rep 30: 1920-1928, 2013.

4. Baek D, Villén J, Shin C, Camargo FD, Gygi SP and Bartel DP: The impact of microRNAs on protein output. Nature 455: 64-71, 2008.

5. Wang YL, Wu S, Jiang B, Yin FF, Zheng SS and Hou SC: Role of microRNAs in prostate cancer pathogenesis. Clin Genitourin Cancer 13: 261-270, 2015

6. Bonci D, Coppola V, Musumeci M, Addario A, Giuffrida R, Memeo L, D'Urso L, Pagliuca A, Biffoni M, Labbaye C, et al: The miR-15a-miR-16-1 cluster controls prostate cancer by targeting multiple oncogenic activities. Nat Med 14: 1271-1277, 2008.

7. Wu Z, He B, He J and Mao X: Upregulation of miR-153 promotes cell proliferation via downregulation of the PTEN tumor suppressor gene in human prostate cancer. Prostate 73: 596-604, 2013.

8. Lewis H, Lance R, Troyer D, Beydoun H, Hadley M, Orians J, Benzine T, Madric K, Semmes OJ, Drake R, et al: miR-888 is an expressed prostatic secretions-derived microRNA that promotes prostate cell growth and migration. Cell Cycle 13: 227-239, 2014. 
9. Verdoodt B, Neid M, Vogt M, Kuhn V, Liffers ST, Palisaar RJ, Noldus J, Tannapfel A and Mirmohammadsadegh A: MicroRNA-205, a novel regulator of the anti-apoptotic protein $\mathrm{Bcl} 2$, is downregulated in prostate cancer. Int J Oncol 43: 307-314, 2013.

10. Ren D, Wang M, Guo W, Huang S, Wang Z, Zhao X, Du H, Song L and Peng X: Double-negative feedback loop between ZEB2 and miR-145 regulates epithelial-mesenchymal transition and stem cell properties in prostate cancer cells. Cell Tissue Res 358: 763-778, 2014.

11. Josson S, Gururajan M, Sung SY, Hu P, Shao C, Zhau HE, Liu C, Lichterman J, Duan P, Li Q, et al: Stromal fibroblast-derived miR-409 promotes epithelial-to-mesenchymal transition and prostate tumorigenesis. Oncogene 34: 2690-2699, 2015.

12. Rajendiran S, Parwani AV, Hare RJ, Dasgupta S, Roby RK and Vishwanatha JK: MicroRNA-940 suppresses prostate cancer migration and invasion by regulating MIEN1. Mol Cancer 13: $250,2014$.

13. Sun Q, Zhao X, Liu X, Wang Y, Huang J, Jiang B, Chen Q and Yu J: miR-146a functions as a tumor suppressor in prostate cancer by targeting Rac1. Prostate 74: 1613-1621, 2014.

14. Dubovenko A, Serebryiskaya T, Nikolsky Y, Nikolskaya T, Perlina A, JeBailey L, Bureeva S, Katta S, Srivastava S, Dobi A, et al: Reconstitution of the ERG gene expression network reveals new biomarkers and therapeutic targets in ERG positive prostate tumors. J Cancer 6: 490-501, 2015.

15. Qiu J, Gao Z and Shima H: Growth of human prostate cancer cells is significantly suppressed in vitro with sodium butyrate through apoptosis. Oncol Rep 27: 160-167, 2012.

16. Shi C, Yu L, Yang F, Yan J and Zeng H: A novel organoselenium compound induces cell cycle arrest and apoptosis in prostate cancer cell lines. Biochem Biophys Res Commun 309: 578-583, 2003.

17. Li X, Gong X, Chen J, Zhang J, Sun J and Guo M: miR-340 inhibits glioblastoma cell proliferation by suppressing CDK6, cyclin-D1 and cyclin-D2. Biochem Biophys Res Commun 460: 670-677, 2015.

18. Fernandez S, Risolino M, Mandia N, Talotta F, Soini Y, Incoronato M, Condorelli G, Banfi S and Verde P: miR-340 inhibits tumor cell proliferation and induces apoptosis by targeting multiple negative regulators of p27 in non-small cell lung cancer. Oncogene 34: 3240-3250, 2015.

19. Poenitzsch Strong AM, Setaluri V and Spiegelman VS: MicroRNA-340 as a modulator of RAS-RAF-MAPK signaling in melanoma. Arch Biochem Biophys 563:118-24, 2014.

20. Yu X, Luo L, Wu Y, Yu X, Liu Y, Yu X, Zhao X, Zhang X, Cui L, Ye G, et al: Gastric juice miR-129 as a potential biomarker for screening gastric cancer. Med Oncol 30: 365, 2013.

21. Wang Y, Jia LS, Yuan W, Wu Z, Wang HB, Xu T, Sun JC, Cheng KF and Shi JG: Low miR-34a and miR-192 are associated with unfavorable prognosis in patients suffering from osteosarcoma. Am J Transl Res 7: 111-119, 2015.

22. Ma L, Reinhardt F, Pan E, Soutschek J, Bhat B, Marcusson EG Teruya-Feldstein J, Bell GW and Weinberg RA: Therapeutic silencing of miR-10b inhibits metastasis in a mouse mammary tumor model. Nat Biotechnol 28: 341-347, 2010.

23. Gordanpour A, Nam RK, Sugar L and Seth A: MicroRNAs in prostate cancer: From biomarkers to molecularly-based therapeutics. Prostate Cancer Prostatic Dis 15: 314-319, 2012.

24. Bhatnagar N, Li X, Padi SK, Zhang Q, Tang MS and Guo B Downregulation of miR-205 and miR-31 confers resistance to chemotherapy-induced apoptosis in prostate cancer cells. Cell Death Dis 1: e105, 2010.
25. Li X, Wan X, Chen H, Yang S, Liu Y, Mo W, Meng D, Du W, Huang Y, Wu H, et al: Identification of miR-133b and RB1CC1 as independent predictors for biochemical recurrence and potential therapeutic targets for prostate cancer. Clin Cancer Res 20: 2312-2325, 2014

26. Wu ZS, Wu Q, Wang CQ, Wang XN, Huang J, Zhao JJ, Mao SS, Zhang GH, Xu XC and Zhang N: miR-340 inhibition of breast cancer cell migration and invasion through targeting of oncoprotein c-Met. Cancer 117: 2842-2852, 2011.

27. Zhou X, Wei M and Wang W: MicroRNA-340 suppresses osteosarcoma tumor growth and metastasis by directly targeting ROCK1. Biochem Biophys Res Commun 437: 653-658, 2013.

28. Honda R, Tanaka H and Yasuda H: Oncoprotein MDM2 is a ubiquitin ligase E3 for tumor suppressor p53. FEBS Lett 420: 25-27, 1997.

29. Montes de Oca Luna R, Wagner DS and Lozano G: Rescue of early embryonic lethality in mdm2-deficient mice by deletion of p53. Nature 378: 203-206, 1995.

30. Chen J, Wu X, Lin J and Levine AJ: mdm-2 inhibits the G1 arrest and apoptosis functions of the $\mathrm{p} 53$ tumor suppressor protein. Mol Cell Biol 16: 2445-2452, 1996.

31. Rayburn E, Zhang R, He J and Wang H: MDM2 and human malignancies: Expression, clinical pathology, prognostic markers, and implications for chemotherapy. Curr Cancer Drug Targets 5: 27-41, 2005.

32. Fortunato O, Boeri M, Moro M, Verri C, Mensah M, Conte D, Caleca L, Roz L, Pastorino U and Sozzi G: mir-660 is downregulated in lung cancer patients and its replacement inhibits lung tumorigenesis by targeting MDM2-p53 interaction. Cell Death Dis 5: e1564, 2014.

33. Leite KR, Franco MF, Srougi M, Nesrallah LJ, Nesrallah A, Bevilacqua RG, Darini E, Carvalho CM, Meirelles MI, Santana I, et al: Abnormal expression of MDM2 in prostate carcinoma. Mod Pathol 14: 428-436, 2001.

34. Zhang Z, Li M, Wang H, Agrawal S and Zhang R: Antisense therapy targeting MDM2 oncogene in prostate cancer: Effects on proliferation, apoptosis, multiple gene expression, and chemotherapy. Proc Natl Acad Sci USA 100: 11636-11641, 2003.

35. Dar AA, Majid S, Rittsteuer C, de Semir D, Bezrookove V, Tong S, Nosrati M, Sagebiel R, Miller JR III and Kashani-Sabet M: The role of miR-18b in MDM2-p53 pathway signaling and melanoma progression. J Natl Cancer Inst 105: 433-442, 2013.

36. Tovar C, Rosinski J, Filipovic Z, Higgins B, Kolinsky K, Hilton H, Zhao X, Vu BT, Qing W, Packman K, et al: Small-molecule MDM2 antagonists reveal aberrant p53 signaling in cancer: Implications for therapy. Proc Natl Acad Sci USA 103: 1888-1893, 2006.

37. Vassilev LT, Vu BT, Graves B, Carvajal D, Podlaski F, Filipovic Z, Kong N, Kammlott U, Lukacs C, Klein C, et al: In vivo activation of the p53 pathway by small-molecule antagonists of MDM2. Science 303: 844-848, 2004

38. Allen JG, Bourbeau MP, Wohlhieter GE, Bartberger MD, Michelsen K, Hungate R, Gadwood RC, Gaston RD, Evans B, Mann LW, et al: Discovery and optimization of chromenotriazolopyrimidines as potent inhibitors of the mouse double minute 2-tumor protein 53 protein-protein interaction. J Med Chem 52: 7044-7053, 2009. 